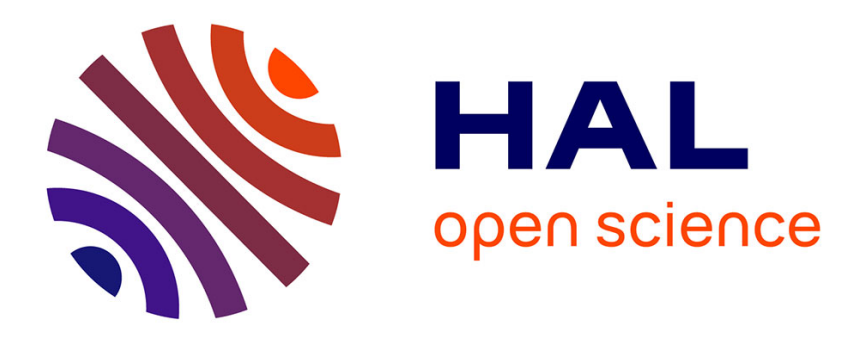

\title{
A Mobile Computer Aided System for Optic Nerve Head Detection
}

\author{
Yaroub Elloumi, Mohamed Akil, Nasser Kehtarnavaz
}

\section{To cite this version:}

Yaroub Elloumi, Mohamed Akil, Nasser Kehtarnavaz. A Mobile Computer Aided System for Optic Nerve Head Detection. Computer Methods and Programs in Biomedicine, 2018, 10.1016/j.cmpb.2018.05.004 . hal-01796762

HAL Id: hal-01796762

https://hal.science/hal-01796762

Submitted on 22 May 2018

HAL is a multi-disciplinary open access archive for the deposit and dissemination of scientific research documents, whether they are published or not. The documents may come from teaching and research institutions in France or abroad, or from public or private research centers.
L'archive ouverte pluridisciplinaire HAL, est destinée au dépôt et à la diffusion de documents scientifiques de niveau recherche, publiés ou non, émanant des établissements d'enseignement et de recherche français ou étrangers, des laboratoires publics ou privés. 


\title{
A Mobile Computer Aided System for Optic Nerve Head Detection
}

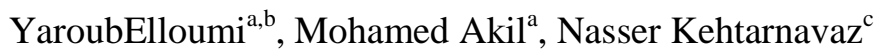 \\ ${ }^{a}$ Gaspard Monge Computer Science Laboratory, ESIEE-Paris, University Paris-Est Marne-la-Vallée, France. \\ ${ }^{\mathrm{b}}$ Medical Technology and Image Processing Laboratory, Faculty of medicine, University of Monastir, Tunisia. \\ ${ }^{c}$ Department of Electrical and Computer Engineering, University of Texas at Dallas, Richardson, TX 75080 USA.
}

\begin{abstract}
:
Background and objective: The detection of optic nerve head $(\mathrm{ONH})$ in retinal fundus images plays a key role in identifying Diabetic Retinopathy (DR) as well as other abnormal conditions in eye examinations. This paper presents a method and its associated software towards the development of an Android smartphone app based on a previously developed $\mathrm{ONH}$ detection algorithm. The development of this app and the use of the D-Eye lens which can be snapped onto a smartphone provide a mobile and cost-effective computer-aided diagnosis (CAD) system in ophthalmology. In particular, this CAD system would allow eye examination to be conducted in remote locations with limited access to clinical facilities.

Methods: A pre-processing step is first carried out to enable the ONH detection on the smartphone platform. Then, the optimization steps taken to run the algorithm in a computationally and memory efficient manner on the smartphone platform is discussed.

Results: The smartphone code of the ONH detection algorithm was applied to the STARE and DRIVE databases resulting in about $96 \%$ and $100 \%$ detection rates, respectively, with an average execution time of about $2 \mathrm{~s}$ and $1.3 \mathrm{~s}$. In addition, two other databases captured by the D-Eye and iExaminer snap-on lenses for smartphones were considered resulting in about $93 \%$ and $91 \%$ detection rates, respectively, with an average execution time of about $2.7 \mathrm{~s}$ and $2.2 \mathrm{~s}$, respectively.
\end{abstract}

Index Terms-Optic Nerve Head detection, smartphone-based CAD in ophthalmology, fundus image processing, Radon Transform.

\section{Introduction}

Mobile platforms, in particular smartphones, are evolving continuously in terms of processing power, storage capacity and captured image quality. It has now become possible to run computationally intensive image processing algorithms, such as face detection and recognition, object identification and tracking, on smartphones. In the medical area, smartphones are now being used for Computer-Aided-System (CAS) of physical activity detection (Liang-Hsuan et al., 2017), neurology performance evaluation (Vianello et al., 2017), etc.; and for Computer-Aided-Diagnosis (CAD) systems including electroencephalogram EEG (Von Luhmann et al., 2016) and electrocardiograph (ECG) (Zhang and Lian, 2009; Arunan et al., 2016). In this paper, we are focusing on a smartphonebased CAD system for retinopathy. Such systems are already known to provide ophthalmologists with a non-labor intensive approach for the detection of abnormalities in retinal fundus images. Another advantage of these systems is their capability to allow eye examination in remote locations with limited access to clinical facilities. The work described in (Bourouis et al., 2014) implements a diabetic feature detection approach proposed in (Gardner et al., 1996), on Android smartphones. The app is compared with retinograph-captured fundus images where the detection accuracy is about $87 \%$.

The localization of Optic Nerve Head (ONH) plays a key role towards identifying conditions such as Diabetic Retinopathy (DR) (Gagnon et al., 2001; Tolias and Panas, 1998; Gupta et al., 2017). ONH appears as a yellowish region in a color retinal fundus image (see Fig.1). The main characteristic of $\mathrm{ONH}$ is its rapid intensity variations due to dark blood vessels that appear in its vicinity. Three characteristics of $\mathrm{ONH}$ have been utilized in the literature to localize it. These characteristics include: (1) appearance as a bright disk nearly $1600 \mu \mathrm{m}$ in diameter; (2) presence of arteries leaving from and veins entering it; and (3) diverging blood vessels from it. As noted in (Kochner et al., 1998), the detection of $\mathrm{ONH}$ is a challenging task due to the discontinuity of its boundary caused by large vessels and its considerable color and intensity variations.

There are many algorithms in the literature that determine the location of ONH or its boundary. In (Shahri et al., 2014), an overview of the existing algorithms was provided and a computationally efficient algorithm in comparison with the existing algorithms was proposed. The algorithm in (Shahri et al., 2014) employed Radon Transform (RT) to localize ONH based on its brightness and roundness. RT processing was applied to overlapped sub-images in order to detect the subimage containing the ONH. RT was applied using different orientations with respect to the $\mathrm{ONH}$ circular shape. It basically involved computing transforms of intensity projections through sub-images. The processing was repeated for overlapping sub-images and different projection orientations. Besides its high computational cost, the RT computation increases when projections along tilted orientations are considered due to an increase in the amount of memory accesses.

This paper proposes a mobile CAD system in ophthalmology that insures locating the fundus on smartphone-captured image and then detecting $\mathrm{ONH}$ using the RT-based ONH detection algorithmreported in (Shahri et al., 2014). The paper discusses the optimization of the RT computation for the purpose of running it as an app on smartphone platforms. Adecomposition is performed to enhance the efficiency of the RT computation by first 
performing RT projection by an angle plane-rotation, followed by orthogonal RT projection that is similar to a line matrix sum. Furthermore, the roundness of ONH is used to reduce the plane-rotation processing time by considering orthogonal projections applied to the same rotated sub-image. This has allowed using the OpenCV library. All the computational steps taken are then implemented on Android smartphones.

The rest of this paper is organized as follows: Section2 provides an overview of the computationally efficient RTbased $\mathrm{ONH}$ detection algorithm. Section 3 discusses the computational issues of this ONH detection algorithm. Section 4 proposes the steps taken to improve the computational efficacy ofthe RT-based ONH detection algorithm. The use of OpenCV and the Android smartphone implementation are presented in section 5. The results obtained in terms of the performance, the computational efficiency and the validation of the developed smartphone app are covered in section 6 followed by discussion in section 7. The paper is then concluded in section 8 .

\section{Overview of Computationally Efficient ONH Detection Algorithm Based on Radon Transform}

The ONH detection algorithm developed in (Shahri et al., 2014) is based on Radon Transform (RT). In this algorithm, the fundus image is partitioned into overlapping blocks or subimages to which RT is applied. The Radon space is then explored several times to identify the ONH.The processing pipeline of the algorithm is illustrated in Fig.1.

First of all, the fundus area is separated from the background with the help of a fundus mask which is either available or can be generated by using the intensity channel. Then, the fundus area is partitioned into sub-images or sliding windows for the detection of the $\mathrm{ONH}$ as illustrated in Fig.2. The approximate size of the $\mathrm{ONH}$ is used to determine the size of the sub-image or sliding window, denoted by $n$. Another

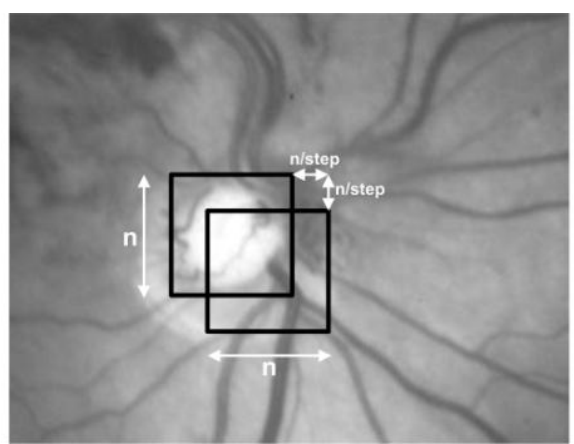

Fig. 2.Window size and overlapping ratio (n, step) in retinal fundus image parameter that has influence on the outcome is the windows shifting ratio which is named step. In (Shahri et al., 2014), it is discussed how to set these parameters.

Next, RT is applied to the sub-images as follows:

$f_{\theta}(s)=\int_{-\infty-\infty}^{+\infty} \int_{-\infty}^{+\infty} f(x, y) \cdot \delta(s-x \cdot \cos (\theta)-y \cdot \sin (\theta)) d x \cdot d y(1)$

where $\theta$ and $\mathrm{s}$ indicate the direction and line parameter, respectively. This equation corresponds to a single projection where the Dirac function $\delta$ denotes the path of the line integral. The projection $\check{\mathrm{f}}_{\theta}(\mathrm{s})$ is interpreted as the onedimensional function $\breve{\mathrm{f}}_{\theta}(\mathrm{s})$ of a single variable $\mathrm{s}$ with $\theta$ as a parameter. RT is able to transform a pattern to a line in the Radon space allowing the pattern to be easily distinguished from other patterns.

RT is then applied to all sub-images along $\omega=12$ directions from $0^{\circ}$ to $165^{\circ}$ degrees with $15^{\circ}$ degrees increment. An $\mathrm{ONH}$ location candidate in a sub-image is associated with a peak in the Radon space. At this stage, all the sub-images which have a peak higher than a threshold are considered to be candidates containing the $\mathrm{ONH}$, such as subimages $\mathrm{Y}$ and $\mathrm{Z}$ shown in Fig.1. In (Shahri et al., 2014), it is discussed how this threshold is selected. The ONH candidates

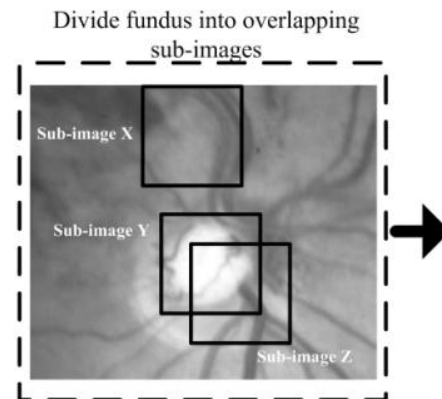

$\mathrm{L}------$

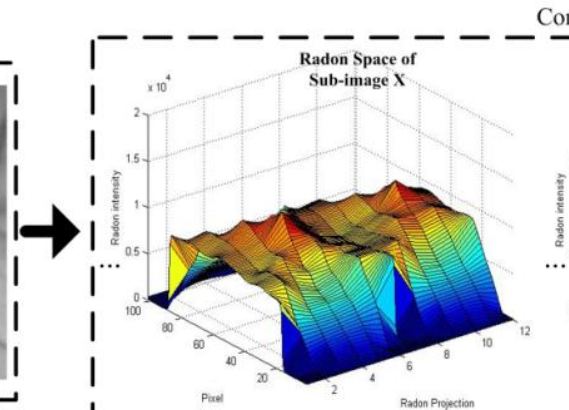

Compute Radon Space for each sub-image
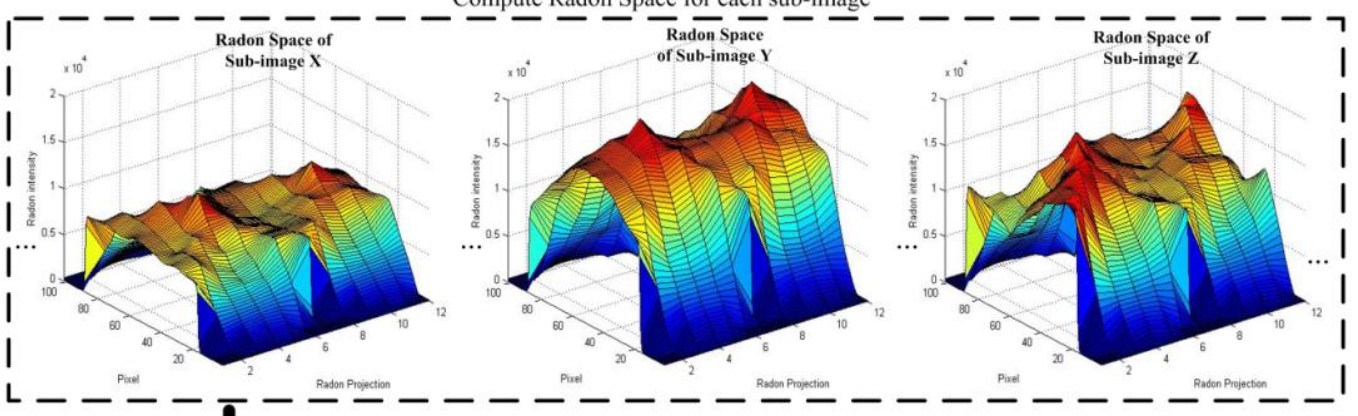

Select sub-image candidates
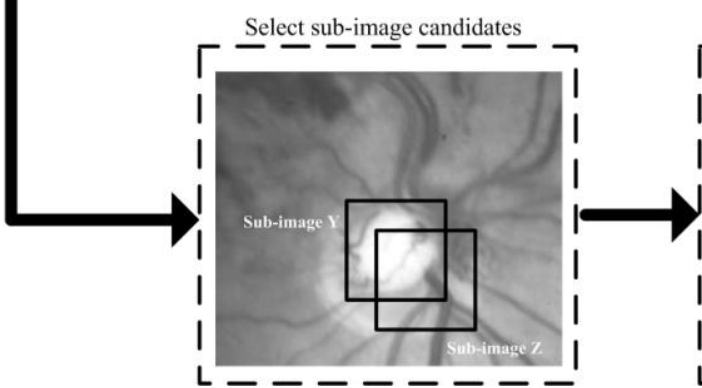

Fig. 1. Processing pipeline of the $\mathrm{ONH}$ detection algorithm

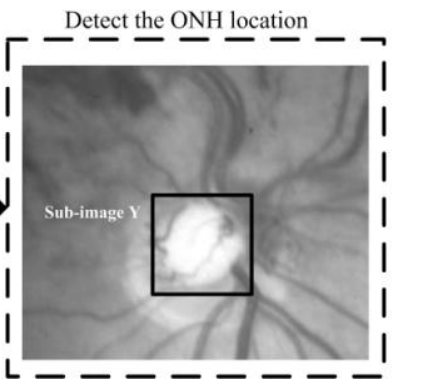


are then refined to find the final $\mathrm{ONH}$ location. The refinement is done by using the roundness of $\mathrm{ONH}$, which prevents the related profiles to differ from each other, to a certain extent.Therefore, a mean vector of the Radon space $\bar{f}$ is computed as noted in (2):

$$
\bar{f}(s)=\frac{1}{\omega} \sum_{\theta=1}^{\omega} f_{\theta}(s)(2)
$$

where $\breve{\mathrm{f}}_{\theta}(\mathrm{s})$ denotes the $(\mathrm{s}, \theta)$ th component of the Radon space, $s$ and $\theta$ indicate the dimensions of the Radon space. Then, the mean square error (MSE) between the $\omega$ projections, as computed in (3), is used as a similarity measure to identify the ONH location.

$$
M S E=\frac{1}{s . \omega} \sum_{s=1}^{s} \sum_{\theta=1}^{\omega}\left(f_{\theta}(s)-\bar{f}(s)\right)^{2}(3)
$$

The $\mathrm{ONH}$ is located by the sub-image exhibiting the least MSE among all the ONH candidates. As illustrated in Fig.1, sub-image Y leads to a Radon space having similar curvatures in terms of Radon projections, compared to sub-image $\mathrm{Z}$. The least MSE corresponds to the ONH location.

All the ONH detection steps are illustrated in the flowchart shown in Fig.3. The fundus area is detected from the fundus image blue component using a binary mask. Then, the RT is applied and the peak value is extracted for each sub-image. All RT peaks are then examined in order to set a threshold.All the

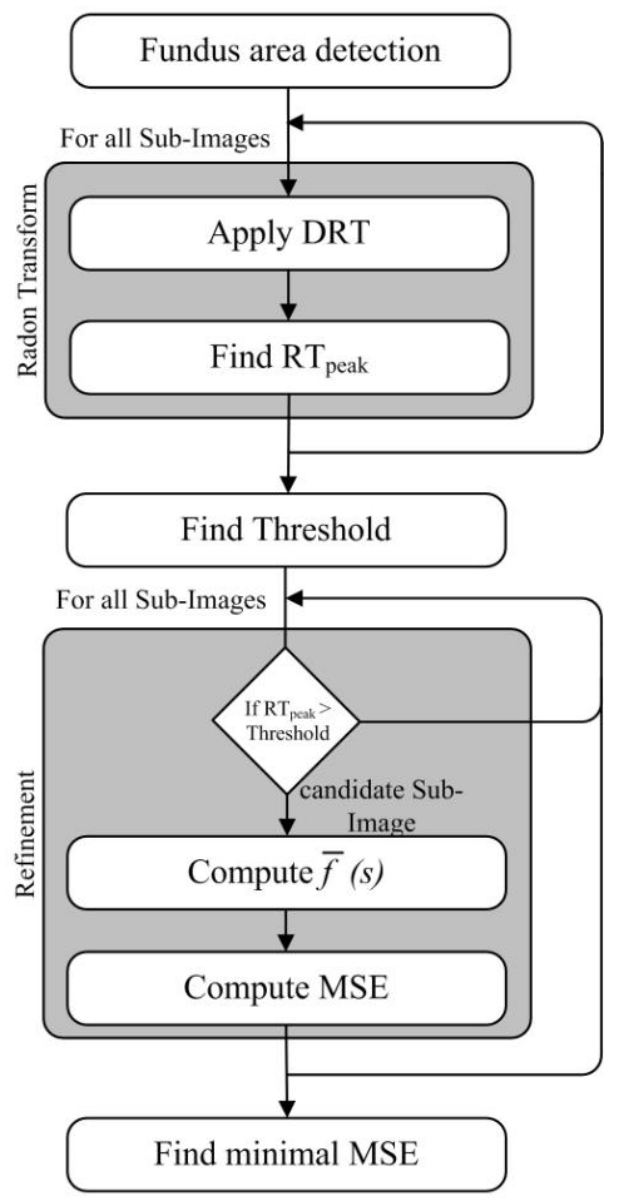

Fig.3.Flowchart of RT-based ONH detection algorithm sub-images with peaks exceeding the threshold are considered to be ONH candidates for which $\bar{f}(s)$ and MSE are computed. The ONH is located in the sub-image having the minimum MSE. In (Shahri et al., 2014), this ONH detection algorithm was shown to achieve the detection accuracies of $100 \%$ and 96.3\% when applied to the DRIVE and STARE database images, respectively.

\section{Analysis of ONH Detection Algorithm for Smartphone APP Development}

\subsection{Computational Complexity Analysis of ONH Detection Algorithm}

The computational complexity of ONH detection was stated in (Shahri et al., 2014). For an input image of size $\mathrm{M} \times \mathrm{N}$, the fundus area detection is of complexity $O(\mathrm{NM})$. The complexity of the refinement component is $O(3 \omega \mathrm{nc})$, where c indicates the number of $\mathrm{ONH}$ candidates, $\mathrm{n}$ the sub-image size and $\omega$ the RT projection number. A single RT projection involves covering all the sub-image pixels and hence has a complexity of $O\left(9 n^{2}\right)$. Each sub-image requires $\omega$ RT projections with predefined angles. RT is applied to all the sub-images thus making the complexity $O\left(\mathrm{MNp}^{2} / \mathrm{n}^{2}\right)$, where $\mathrm{p}$ denotes the window step. Consequently, for an input of size $\mathrm{M} \times \mathrm{N}$ with the window size $\mathrm{n}$ and the step $\mathrm{p}$, the total number of operations involved in the algorithm is $O\left(9 \mathrm{MN} \omega \mathrm{p}^{2}\right)$. For the optimal values of $\mathrm{n}, \omega, \mathrm{c}$ and $\mathrm{p}$ parameters noted in (Shahri et al., 2014), RT requires to be computed more than 1000 times as compared to the fundus area detection and refinement components. In other words, the optimization of RT processing plays the major or key role in the efficiency of the algorithm running on a smartphone platform.

\subsection{Discrete Aspect of Radon Transform}

A Radon projection beam runs in a straight line across an image at an orientation $\theta$ in the $x-y$ plane. A radon projection is a set of parallel beams perpendicular to the line at angle $\theta$ (Nacereddine et al., 2015). Due to the discrete nature of images, Discrete Radon Transform (DRT) as proposed in (Kingston, 2006) is computed. DRT consists of two equations that correspond to projections with $0 \leq \theta \leq \pi / 4$ and projections with $\pi / 4 \leq \theta \leq \pi / 2$. The equation below provides the DRT computation (Dai et al., 2010; Kingston and Svalbe,2003):

$$
\begin{aligned}
& R(s, \theta)=\sum_{0}^{n-1} \sum_{0}^{n-1} I(x, y) \cdot \delta\left(s-x \cdot y_{\theta}+y \cdot x_{\theta}\right) \\
& y_{\theta} \cdot \sin (\theta)=x_{\theta} \cdot \cos (\theta), x_{\theta} \in Z, y_{\theta} \in Z
\end{aligned}
$$

where $I$ is an input sub-image of size $n \times n$ where $n \in N$, $x$ and $\mathrm{y}$ are the pixel indexes in $\mathrm{I}, \delta$ is the Dirac function where $\delta(\mathrm{x})=1$ if $\mathrm{x}=0$ and $\delta(\mathrm{x})=0$ in all other cases, $\mathrm{s} \in$ $\left\{0,1,2, \ldots, N_{\theta-1}\right\}, N_{\theta}=N\left(\left|x_{\theta}\right|+y_{\theta}\right), x_{\theta}$ and $y_{\theta}$ denote the horizontal and vertical distances from the nearest pixels, respectively. The DRT of a sub-image are stored in a $\mathrm{s} \times \mathrm{n}_{\theta}$ matrix, called Radon space, where $s$ denotes the projection line index and $n_{\theta}$ the angle number. 


\subsection{DRT ComputationalComplexity}

DRT requires the processing of several beams for each subimage. As indicated in (4), a RT projection $R(s, \theta)$ requires examining all the sub-image pixels. For each pixel, a Dirac delta computation is required that involves trigonometric functions. The work described in (Kim and Kim, 2007; Schmit and Thomas, 1995) indicates that the index calculation causes a delay due to the memory access latency. Consequently, the index calculation poses an implementation bottleneck that significantly impacts the $\mathrm{ONH}$ detection computation.

Furthermore, all the pixels that cross a beam should be taken into account where their number might reach 2. $\max$ (row, column). For a DRT projection with $\theta=\frac{\pi}{4}$ as depicted in Fig. 4(a), each beam involves accessing 19 pixels for an image size of $10 \times 10$. Thus, the projection angle has also an impact on the computational complexity of processing a beam.

Processing a beam requires accessing non-successive pixels with different indexes in terms of rows and columns. Consider the example of the RT projection illustrated in Fig. 4(a), the pixel row indices and pixel column indices are both varied in the ranges [1..10]. In fact, the memory has several banks; each bank consists of two-dimensional arrays which are referred by its rows and columns (Subramanian et al., 2016; Lee et al., 2013). Due to the image size, pixels are saved in different array rows. Every memory access allows reading one row data per cycle, named buffer. Thus, pixels belonging to different row buffer lead to a pipeline stall. The row switches imply a time that is unavailable (Yin et al., 2016; Yuan et al., 2009) and thus a throughput bottleneck is created. In (Subramanian et al., 2016), it has been shown that reading data from different row buffers leads to a double or triple increase in the memory access latency.

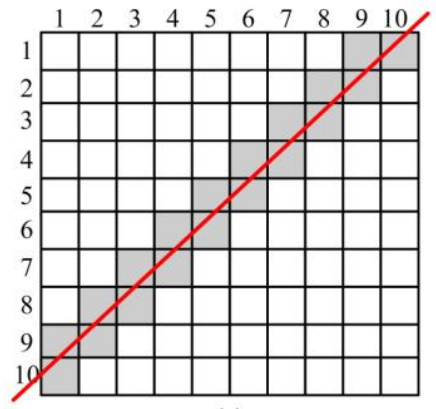

(a)

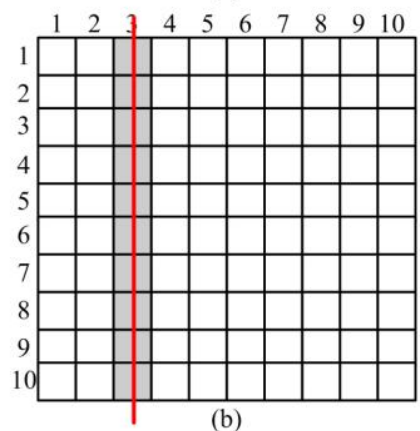

Fig.4. Beam projections: (a)with $\theta=\pi / 4$; (b) with $\theta=2 \pi$

\section{Optimization of RT-Based ONH Detection Algorithm}

\subsection{RT Computation UsingPlane Rotation}

Image rotation is employed in many computer vision applications. The rotation transformation in Cartesian coordinates as noted in (5) can be done by a one-pass approach to determine each rotated point as described in (Ashtari et al., 2015; Nouvel and Rémila, 2006; Chen et al., 2014).

$$
\left(\begin{array}{l}
x_{r} \\
y_{r}
\end{array}\right)=\left(\begin{array}{cc}
\cos (\theta) & -\sin (\theta) \\
\sin (\theta) & \cos (\theta)
\end{array}\right)\left(\begin{array}{l}
x \\
y
\end{array}\right)(5)
$$

where $\theta$ denotes the rotation angle, $(x, y)$ the coordinates of a pixel in the input image, $\left(\mathrm{x}_{\mathrm{r}}, \mathrm{y}_{\mathrm{r}}\right)$ the rotated coordinates of the pixel in the output image, respectively. The rotation given in (5) is of complexity $O\left(6 \mathrm{n}^{2}\right)$ as mentioned in (Kingston, A., $2006)$ for a rotated image of size $n \times n$. The work described in (Yu et al., 2016) proposes an incremental rotation that requires $\mathrm{O}\left(\mathrm{n}^{2}-\log (\mathrm{n})\right)$ operations. A one-pass method for image rotation is also discussed in (Ashtari et al., 2015) which is of complexity $\mathrm{O}(2 \mathrm{n})$ while preserving image quality. Parallel processing implementations of image rotation on hardware platforms have also appeared in the literature, e.g. (Yu et al., 2016; Mazumdar and Mukhopadhyay, 2017).

There are a number of papers that address rotation and RT jointly. In (Svalbe, 2011), it was shown that an exact 2D image rotation can be achieved by re-ordering the elements of RT projections. In (Lidong et al., 2015), the relation between RT and plane-rotation was formulated by applying RT with an angle $\theta$ to a rotated image by angle $\varepsilon$ similar to applying RT with an angle $(\theta+\varepsilon)$, as indicated in $(6)$,

$$
f_{\theta}\left(\operatorname{rot}_{\varepsilon}(s)\right)=\xi_{\theta+\varepsilon}(s)(6)
$$

In fact, using $\theta=2 \pi$ in the RT function shown in (4) implies that Dirac component is equal to $\delta(s-x)$. Hence, each $\mathrm{k}$ beam depends only onx indices. Therefore, the DRT becomes equal to the pixels sum where $y=s$, as indicated in (7). Consequently, RT needs to be performed n times. Fig.4(b) shows an example of a vertical beam where the crossed pixels are indicated by gray color.

$$
R(k, 2 \pi)=\sum_{i=0}^{n-1} I(i, k)(7)
$$

Thus, RT is formulated here with angle as RT with angle $0^{\circ}$ after rotating the image with $\varepsilon$. This property leads to computing RT as noted in (8),

$$
f_{\varepsilon}(s)=f_{0}\left(\operatorname{rot}_{\varepsilon}(s)\right)(8)
$$

Given the computational complexity of (5) and (7), Eq. (8)becomes of complexity $O\left(6 n^{2}+n\right)$ instead of $O\left(9 n^{2}\right)$, which denotes a reduction in the processing time. Furthermore, successive pixel indices are foundfrom minimal row buffers that lead to a reduction of the memory access latency. Moreover, the beam value is computed using the minimum pixel number which is equal to $\max (M, N)$ for an image of size $\mathrm{M} \times \mathrm{N}$. In addition, no index parameters are involved. Thus, applying RT with angle $\theta=2 \pi$ leads to a 


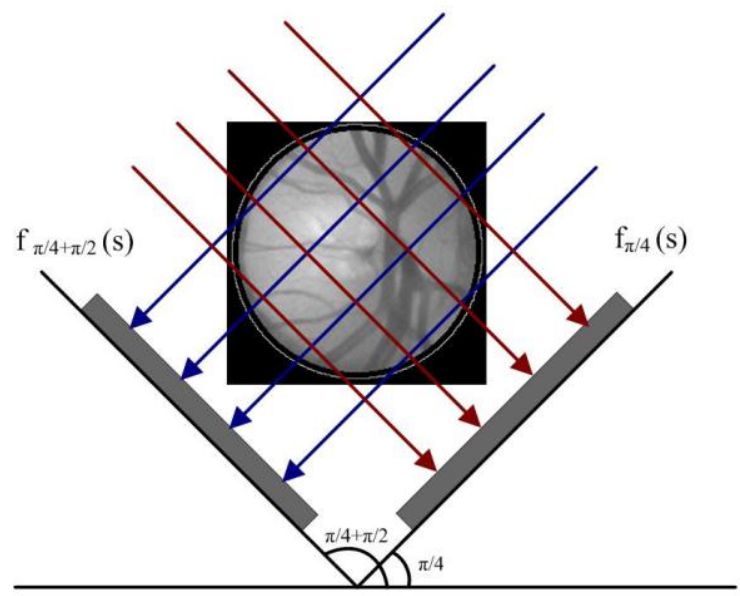

(a)

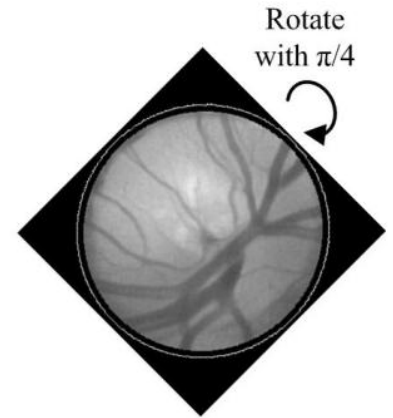

(b)

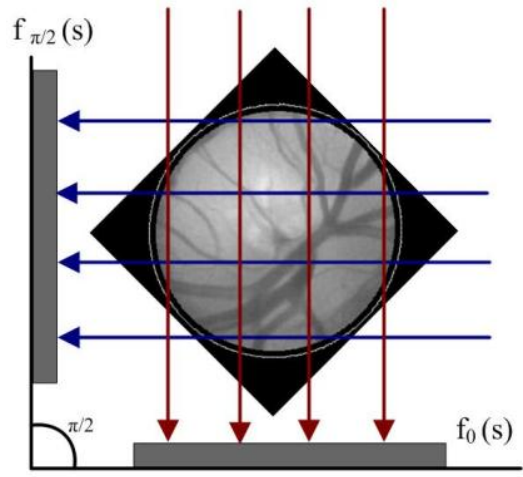

(c)

Fig. 5. Processing RT projections with $\pi / 4$ and3. $\pi / 4$ : (a) initial sub-image; (b) rotation of the sub-image by $\pi / 4$; (c) processing RT projections with 2 . $\pi$ and $\pi / 2$ applied to the rotated sub-image

reduction in the computational complexity of the beam processing and DRT.

\subsection{Merging of RT Projection Processing}

$\mathrm{ONH}$ detection based on RT involves applying RT along different projection angles in order to capture the $\mathrm{ONH}$ pattern in a sub-image. The detection outcome increases with the number of projection angles. The angle range $[0, \pi]$ is equally partitioned in order to have the same projection on both halves of a sub-image. The value chosen are applied in 12 directions from 0 to $165^{\circ}$ in $15^{\circ}$ increments. As a result, for any angle $\theta \in[0, \pi / 2[$, there is an angle $\varphi \in[\pi / 2, \pi[$ where $\varphi=\theta+$ $\pi / 2$, as depicted in Fig. 6. Therefore, angles can be merged based on $(\omega / 2)$ of $(\theta, \varphi)$ angles, where $\omega$ denotes the angle number.

When applying RT by using $\theta=\frac{\pi}{2}+2 \pi$, the Dirac component of RT function is equal to $\delta(s-y)$. Therefore, the DRTbecomes similar to a horizontal projection where each beam is equal to the pixel sum where $\mathrm{x}=\mathrm{s}$, as indicated in (9).

$$
R(k,(\pi / 2)+2 \pi)=\sum_{j=0}^{n-1} I(k, j)(9)
$$

Based on (6), applying RT projection with angle $\theta+\pi / 2$ is similar to applying RT projection with $\pi / 2$ applied to a rotated image with angle $\theta$, as noted in (10). Therefore, the DRT is

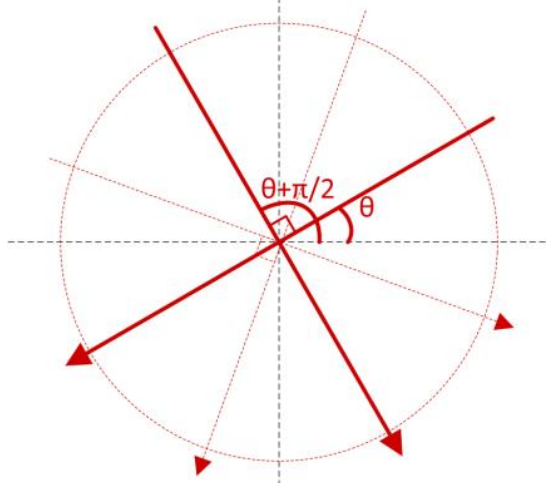

Fig. 6. Orientation of RT projections computed as in (10) which involves row sums thus avoiding the limits mentioned earlier.

$$
f_{\theta+(\pi / 2)}(s)=f_{\pi / 2}\left(\operatorname{rot}_{\theta}(s)\right)(10)
$$

In other words, merging of RT projection processing is done by performing both $\theta$ and $\varphi$ RT projections by rotating a sub-image by $\theta$, followed by RT projection with angle $\theta$ via adding all the rows, while RT projection with angle $\varphi$ is done via adding all the columns. As an example, Fig. 5(a) illustrates the RT projections with angle $\theta=\pi / 4$ and $\varphi=3 . \pi / 4$. First, the sub-image is rotated by $\pi / 4$ as shown in Figure 5(b). Then, $\breve{\mathrm{f}}_{\pi / 4}$ and $\breve{\mathrm{f}}_{3 . \pi / 4}$ are performed, respectively, with horizontal and vertical directions, as illustrated in Fig.5(c).

To preserve accuracy, the plane rotation need to maintain the same pattern size as the intput image. Thus, a rotated subimage of size $n \times n$ is transformed to a matrix of size $m \times m$ where $m=\sqrt{2} . n$ as mentioned in (Svalbe, 2011; Jadhav and Holambe, 2010). This matrix has a dark background in order to avoid getting noise into the RT processing, see Fig. 7(b). In fact, each input sub-image is filtered via a binary mask in order to preserve a circular form with respect to the $\mathrm{ONH}$. Knowing that a circular object remains invariant to a plane rotation, the middle matrix of size $n \times n$ incorporates all the patterns of interest. Therefore, the orthogonal DRT is applied only to the $(n \times n)$ centered portion which has the same size as the input sub-image, as shown in Fig. 7(c). Hence, the DRT complexity remains the same despite the sub-image rotation. In other words, the sub-image plane rotation leads to halving the rotation processing that is performed only wdiv 2 instead of $\omega$ times, while preserving the sub-image size and hence the number of RT beams of $\check{f}_{\theta}$, where $\theta=n \times \pi / 2$ and $n \in N$. Note that such optimization is achieved regardless of the $\omega$ pair projection number.

All the DRT projections are computed and collected in the RT space, as outlined in Algorithm 1. The outermost loop correspond to ( $\omega$ div 2 ) iterations that in turn corresponds to the sub-image rotation. Thereafter, all pixels of the central component are examined in order to compute $\check{\mathrm{f}}_{\theta}$ and $\check{\mathrm{f}}_{\theta+\pi} \pi / 2$ 


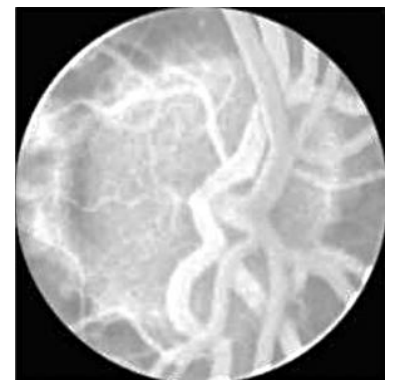

(a)

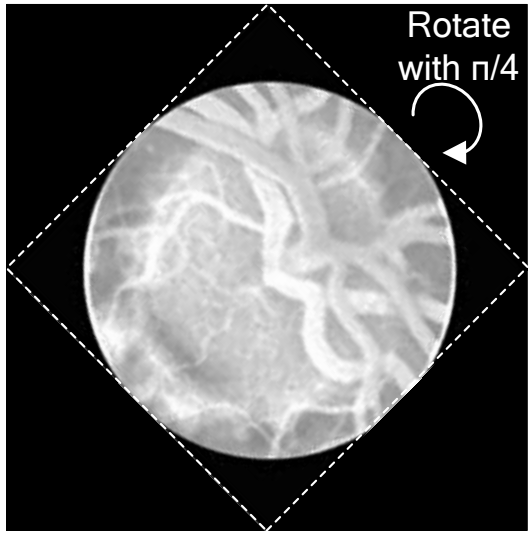

(b)

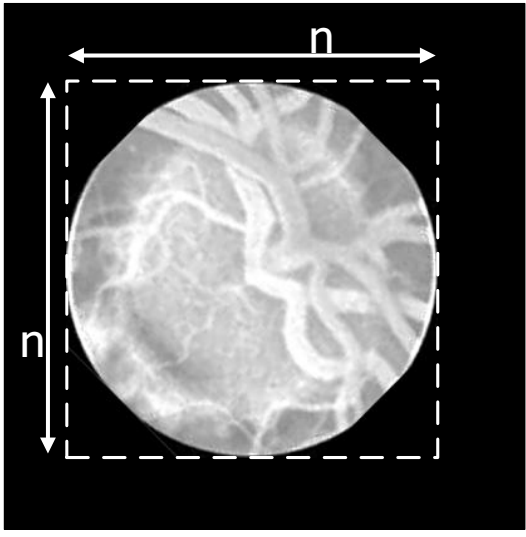

(c)

Fig. 7.Processing RT projections with $\pi / 4$ and 3. $\pi / 4$ : (a) initial sub-image; (b) rotated sub-image by $\pi / 4$ and saved in dark background; (c) the region of interest

\begin{tabular}{l} 
Algorithm 1 :RT space processing \\
\hline $\begin{array}{l}\text { Inputs: sub-image } S I \text { with size }(n \times n) ; \\
\text { vector } A \text { of projection angles where } \operatorname{card}(A)=\omega \\
\text { Outputs:RT space } \check{f}[\omega, n]\end{array}$ \\
\hline Begin \\
For $\theta$ from $A[1]$ to $A[\omega \operatorname{div} 2]$ do \\
Rotate $S I$ on $R S I$ with angle $\theta$ \\
For $k$ from $(\sqrt{2} \cdot n)-(n / 2)$ to $(\sqrt{2} \cdot n)+(n / 2)$ do \\
Forifrom $(\sqrt{2} \cdot n)-(n / 2)$ to $(\sqrt{2} \cdot n)+(n / 2)$ do \\
$\qquad \check{f}[\theta, k] \leftarrow \check{f}[\theta, k]+R S I(k, i)$ \\
$\qquad \check{f}[\theta+\pi / 2, k] \leftarrow \check{f}[\theta+\pi / 2, k]+R S I(i, k)$ \\
End for \\
End for \\
End for \\
End
\end{tabular}

simultaneously. For this purpose, the two innermost loops ensure the browsing of all the image pixels when $\operatorname{RSI}(\mathrm{k}, \mathrm{i})$ is added to $\breve{\mathrm{f}}[\theta, \mathrm{k}]$ and $\mathrm{f}[\theta+\pi / 2, \mathrm{k}]$. The innermost loops need to be computed $\left(4 . n^{2}\right)$ times. Hence, an iteration of the outermost loop is performed $\left(4 . n^{2}+2 . n\right)$ times. Consequently, the RT spacecomplexity becomes $\left(\left(2 . \mathrm{n}^{2}+\right.\right.$ n). $\omega$ ) instead of $\left(9 . n^{2} . \omega\right)$ as described in (Shahri et al., 2014).

\section{Smartphone-Based ONH Detection CAD System}

Smartphones are increasingly being used in medical applications. The number of individuals using smartphones was estimated to be 1000 million in 2012, and is expected to reach 2500 million by 2020 (Harwood et al., 2017). Smartphones have dramatically improved in terms of processing power, storage capacity and connectivity. In ophthalmology, several mobile lens units have been developed for capturing retinal fundus images (Besenczi et al., 2015; Blanckenberg et al., 2011). In particular, the lens unit in (Sharma, 2017; Russo et al., 2015) named D-Eye is designed to be easily snapped onto a smartphone.

The main thrust here is to provide a smartphone-based Computer-Aided-Diagnostic (CAD) system for the detection of $\mathrm{ONH}$. The smartphone-captured fundus images express the same characteristics as of retinograph-captured ones. This will allow the above processing pipeline to be run on smartphones as apps.

\subsection{Fundus Location in Smartphone-Captured Images}

Retinograph-captured images are characterized by a sufficient contrast level that allows distinguishing between optic disc and blood vessels from eye fundus. This characteristic allows a higher accuracy performance when image processing is performed based on brightness, as discussed in (Shahri et al., 2014). Moreover, due to the fixed distance to the camera lens, the fundus always occupies more or less the same image area. A static binary mask is then applied to attribute dark color to the fundus background.

Fundus images captured by smartphones via D-Eye have lower quality than the ones captured with retinographs. Smartphone captured fundus images often have a nonbalanced contrast. A light leakage normally occurs which leads to fundus and background having a similar contrast. To avoid this problem, the Contrast Limited Adaptive Histogram Equalization (CLAHE) approach in (Lidong et al., 2015) is applied here to the blue component of the fundus image, as illustrated inFig. 8 .

The eye fundus size and location differ from one image to another due to the handholding of a smartphone. Thus, no predefined binary mask can be applied to outline the fundus. Here, the roundness is used to outline the fundus. For this purpose, the image is dilated in order to dispose blood vessels. Then, the circular Hough transform is applied to detect the circular pattern of the fundus. A radius range is assigned to avoid detecting the ONH instead of the fundus. This way, the radius and center indices represent the parameters of the binary mask applied to outline the fundus, see Fig.8.

\subsection{Mobile ONH Detection CAD System}

The developed mobile ONH detection CAD system consists of the lens described in (Sharma, 2017; Russo et al., 2015). 


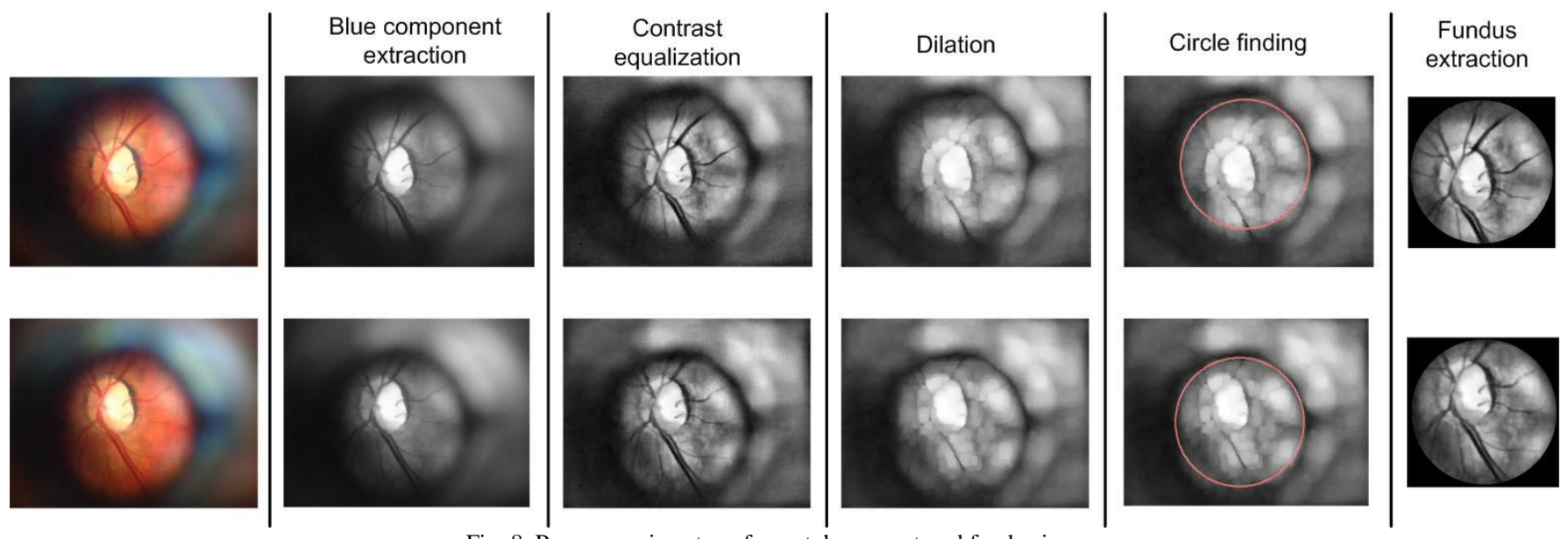

Fig. 8. Pre-processing step of smartphone-captured fundus image

The smartphone is then used to capture and process the fundus image, and shows the $\mathrm{ONH}$ detection outcome on its screen, as illustrated in Fig. 9. To achieve a comparable detection accuracy reported in (Shahri et al., 2014), the pre-processing step described in the previous subsection is applied to outline the fundus location and then the optimized $\mathrm{ONH}$ algorithm described in section IV is executed.

The OpenCV library is used in the above processing steps in order to guarantee computationally efficient execution. The

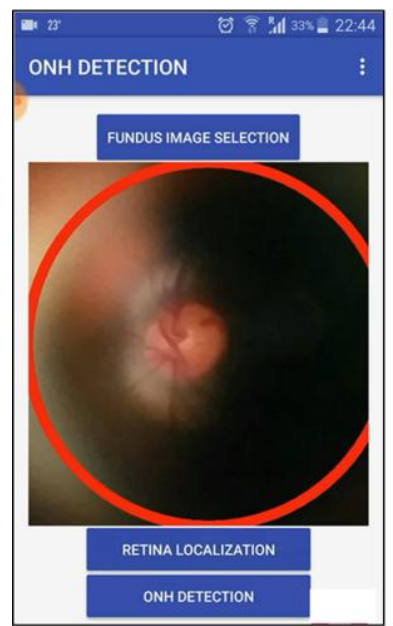

(a)

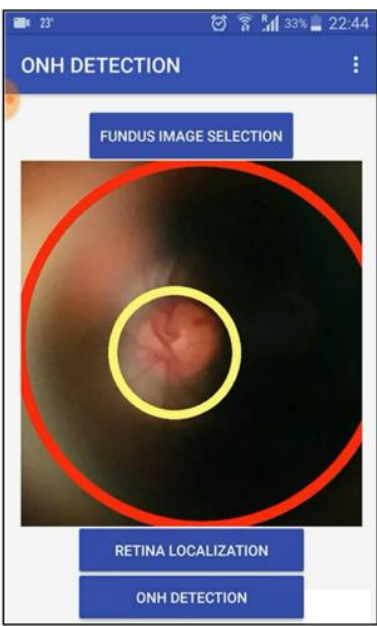

(b)
Fig. 9. Smartphone graphical interface: (a) retina localization; (b) $\mathrm{ONH}$ detection

entire system is implemented on an Android smartphone where the processing time takes about 2 s. A video clip of the developed CAD system for $\mathrm{ONH}$ detection appears at (Elloumi et al., 2017a). The developed CAD system also works when using the iExaminer lens (Sharma, 2017) by which better quality fundus images can be captured as reported in (Besenczi et al., 2015).

\subsection{Software Environment}

The implementation was carried out for Android smartphones using the Android Software development kit (SDK). When processing complex operations are written in the Android language Java, they run in many cases slower than the ones written in native $\mathrm{C} / \mathrm{C}++$ code (Sun and Tan,
2014; Sun and Tan, 2014; Lee and WookJeon, 2010; Mitrovic et al., 2016). The use of $C$ allows accessing advanced libraries towards efficient implementations (Harwood and Revell, 2017). Android Native Development Kit (NDK) allows the codes written in $\mathrm{C} / \mathrm{C}++$ to integrate into the Android SDK environment. The Java Native Interface (JNI) is used here which is the programming framework that allows Java codes to embed native $\mathrm{C} / \mathrm{C}++$ codes.

Open Computer Vision (OpenCV) is a widely used open source library for image processing and computer vision written in C++ ((Harwood and Revell, 2017; De Marsico et al., 2014). It provides more than 2500 optimized image processing functions. The coding for the $\mathrm{ONH}$ detection algorithm was done by combining OpenCV and Java programming languages. When the $\mathrm{ONH}$ detection app is run, the OpenCV functions are called from the JNI as modeled by the arrows illustrated in Fig. 10. Therefore, the JNI is then compiled using the NDK software tool. The following steps were taken to develop an Android app using the OpenCV library of image processing functions:

- Importing OpenCV onto a project in the Android environment.

- Configuring the project features by creating dependency with the OpenCV library and by updating the compile and target SDK versions in order to integrate OpenCV syntax in the Android Java code.

- Setting up the JNI framework to ensure that the OpenCV

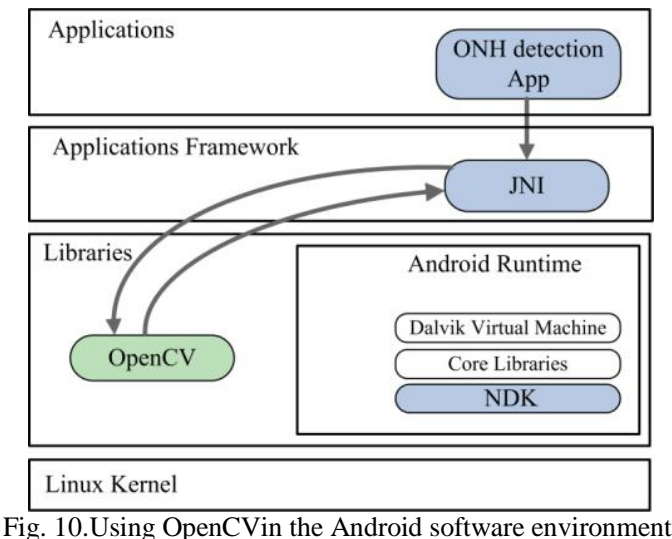


library methods are employed. A "jniLibs" folder is created in the project main folder. The CPU-architecture(s) of the target smartphone device among ARMv5, ARMv7, x86, MIPS, ARMv8, MIPS64 and x86_64 needs to be specified. A free software, called "Native Libs Monitor" would allow identifying the target CPUarchitecture. Then, the folders that correspond to the target CPU-architecture are copied from the OpenCV library to the created jniLibs folder.

- Installing NDK and configuring the project to compile and run the app by modifying the gradle file.

\section{ExperimentalResults}

\subsection{ONH Detection Evaluation}

\subsubsection{Detection Accuracy}

The ONH detection algorithm was evaluated via a desktop implementation done in Matlab and by using two widely used databases of retinal images. The first experiment aimed at investigating the accuracy when the algorithm was run on an Android smartphone. The hardware features of the PC and smartphone used are listed in Table I.

The STARE and DRIVE retinal database images were used for this detection accuracy study. We found that both the smartphone and desktop implementations achieved the same $\mathrm{ONH}$ detection accuracy on the two databases indicating the correct functionality of the smartphone app developed. The accuracy results have been summarized in Table II.

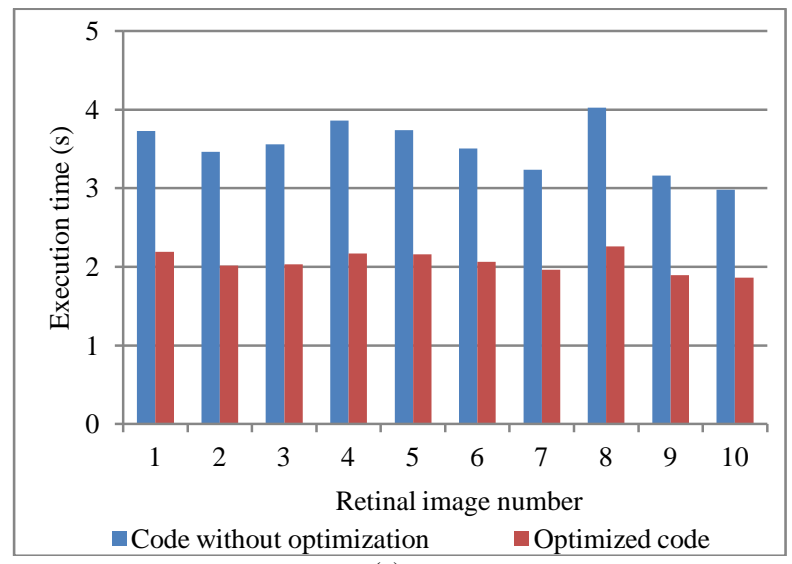

(a)

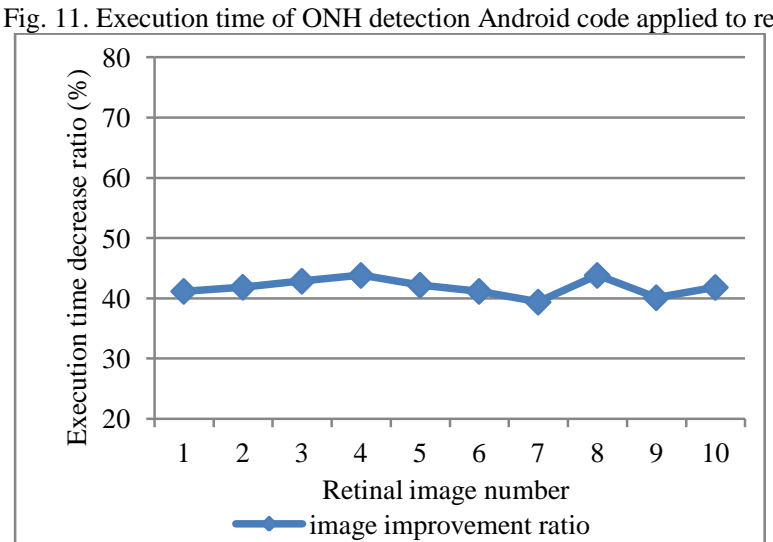

(a) improvement ratio

Fig. 12. Optimization improvement ratio in terms of retinal images: (a) STARE database;

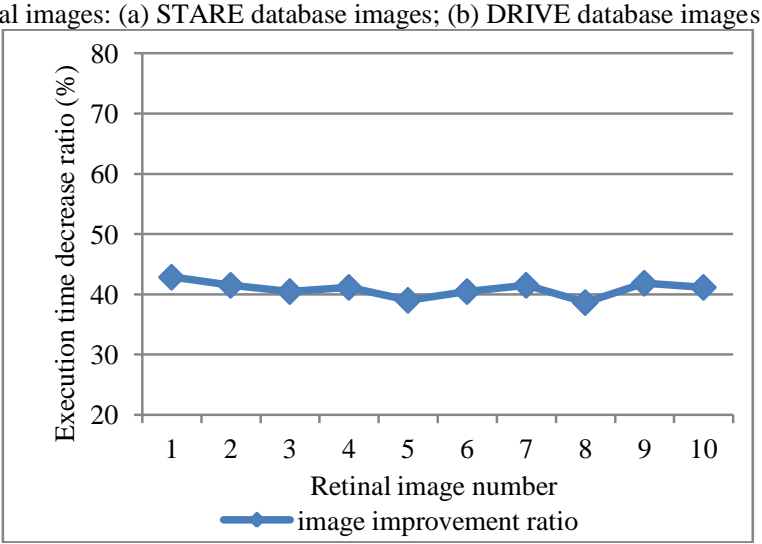

(b)

TABLE I

HARDWARE FEATURES OF IMPLEMENTATION PLATFORM

\begin{tabular}{ccc}
\hline & PC & Mobile device \\
\hline Model & HP ProDesk 400 & Samsung Galaxy S5 \\
\hline CPU architecture & Core i(5) - 4590s & $\begin{array}{c}\text { Qualcomm } \\
\text { MSM8974PRO-AC }\end{array}$ \\
\hline CPU number & 4 cores & 4 cores \\
\hline CPU frequency & 3.30 Ghz & 2.5 Ghz \\
\hline RAM & 8 GB & AnB \\
\hline OS & Win 7 Professional & \\
\hline ONH DETECTION ACCURACY IN TERMS OF RETINAL IMAGE DATABASE \\
\hline Matlab code & Android Code \\
\hline DRARE & $96.3 \%$ & $96.3 \%$ \\
\hline DRIV & $100 \%$ & $100 \%$ \\
\hline
\end{tabular}

\subsubsection{Processing Time Improvement}

We conducted two experiments to examine the execution time. The first experiment examined the optimization steps taken to speed up the detection of $\mathrm{ONH}$ on a smartphone platform. The second experiment compared the $\mathrm{ONH}$ detection between the Android and the desktop codes. We chose 10 images at random for this study from each of the

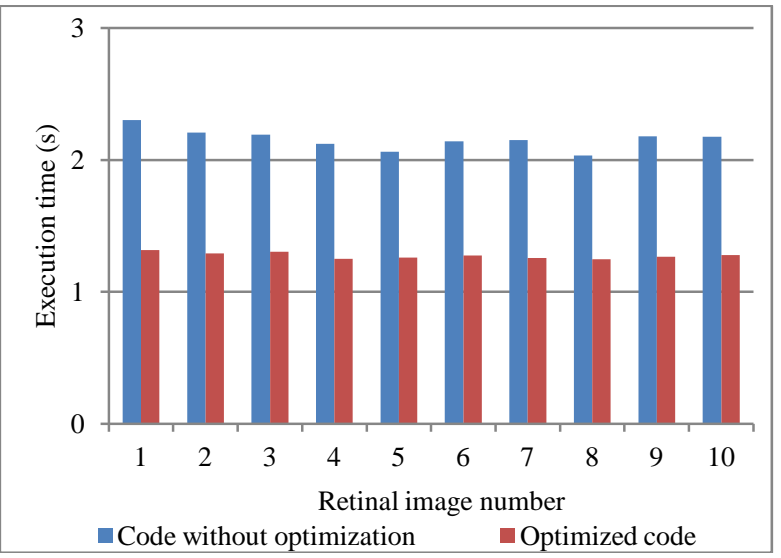

(b)

b) DRIVE database 


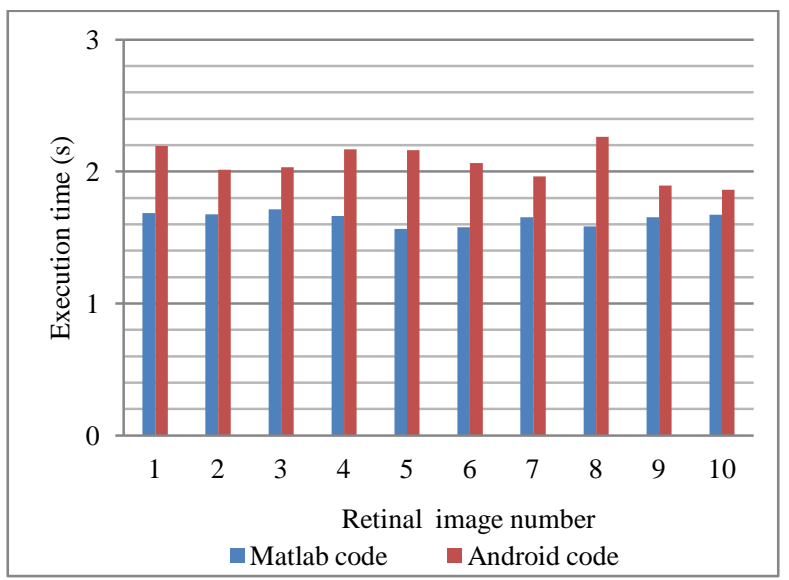

(a)

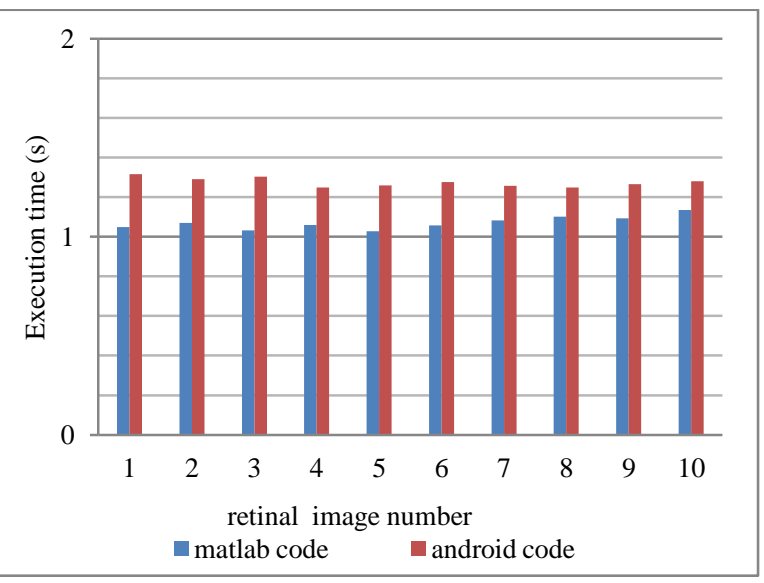

(b)

Fig. 13. Android code execution time of retinal images: (a) STARE database images; (b) DRIVE database images

STARE and DRIVE databases. Fig. 11 shows the execution time for 10 sample images of each database. The average execution time of non-optimized Android code was about 3s, whereas the execution time for the optimized version was about $1.8 \mathrm{~s}$, for the STARE retinal images. This exhibited about $40 \%$ improvement in the execution time. The same $40 \%$ improvement was observed for the DRIVE retinal images. Moreover, the variations in the execution time from STARE DB images to DRIVE DB images were attributed to the number of candidate sub-images that were selected to detect the ONH. It is important to note that the improvement in the execution time was found to be independent from the retinal anatomy, as illustrated in Fig. 12.

We compared the Android codes execution time to the reference Matlab code running on a desktop PC. The results are summarized in Fig.13 exhibiting the execution times for both PC and smartphone implementations. The Android smartphone execution times are found to be higher by about $20 \%$ and $16 \%$ than the Matlab desktop execution times for the STARE and DRIVE database images, respectively.

\subsection{Evaluation of Smartphone-Based CAD System}

To evaluate the developed CAD system for retinal images captured by the D-EYE lens, we considered a D-EYE database

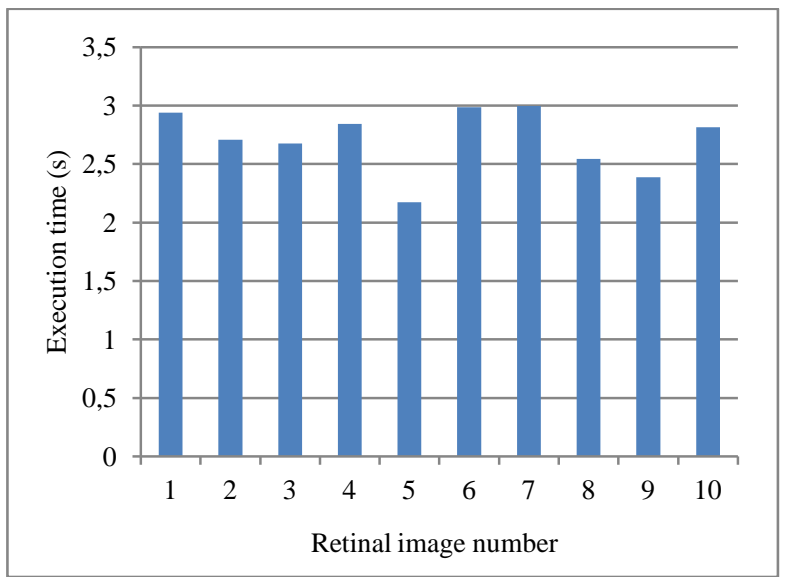

(a)
TABLE III

ONH DETECTION ACCURACY IN TERMS OF RETINAL IMAGE DATABASE

\begin{tabular}{ccc}
\hline & Matlab code & Android Code \\
\hline $\boldsymbol{D}$ - EYE DB & $92.85 \%$ & $92.85 \%$ \\
\hline $\boldsymbol{R}$ IAMP-DB & $91.66 \%$ & $91.66 \%$ \\
\hline
\end{tabular}

consisting of 14 images of size $1200 * 1200$ pixels, which are hosted in (Elloumi et al., 2017b).In addition, the database called "Retinal Image Acquired by Mobile Phone (RIAMP)" in (Besenczi et al., 2015) was used which consisted of 48 fundus images of size $2000 * 1500$ pixels. As noted in Table III, the experiments revealed that the $\mathrm{ONH}$ was localized in about $93 \%$ of the D-EYE database images and in about $91 \%$ of the RIAMP database images.

As shown in Fig. 14, the computational time was also examined using 10 retinal images from both of these databases. The CAD system provided an efficient execution time of about $2.2 \mathrm{~s}$ and $2.7 \mathrm{~s}$ on average, respectively, for the RIAMP and D-EYE database images.

\section{Discussion}

The experimental results indicate that an effective $\mathrm{ONH}$ detection can be achieved based on contrast and roundness

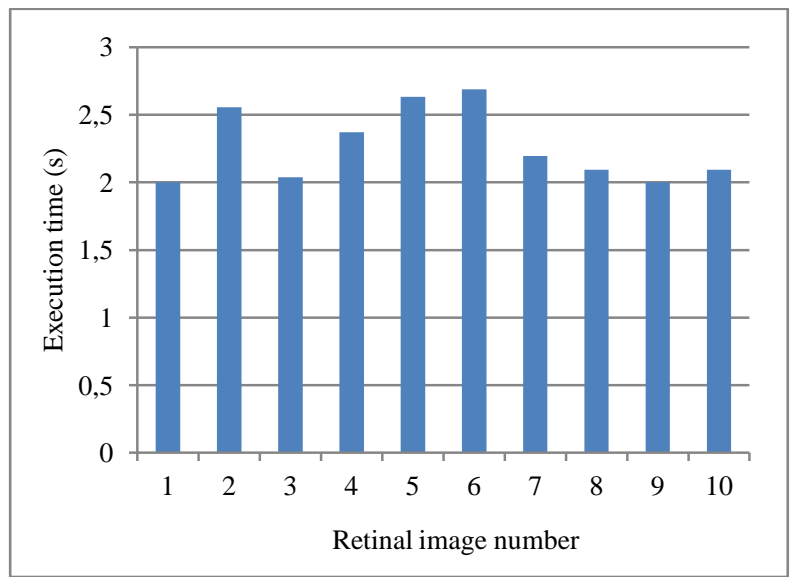

(b)

Fig. 14. CAD system execution time: (a) D-EYE database images; (b) RIAMP database images 
from smartphone-captured fundus images noting that these images are challenging to work with due to the presence of motion blur and non-balanced contrast caused by the handheld holding of smartphones.

Although higher detection as compared to conventional fundus images is achieved for the databases examined, it is worth noting that higher detection is not guaranteed for all possible shapes, colors and contrasts. As future work, we are developing a point spread function estimation approach similar to (Dash and Majhi, 2014) for deblurring and denoising smartphone captured fundus images.

In terms of DR detection, its first stage, called nonproliferative DR, is characterized by emerging different lesions such as hard exudates, microaneurysms and haemorrhages. Hard exudates appear as bright shapes in retinal images (Prentašić and Lončarić, 2016; Zhang et al., 2014). Our CAD system has the potential to detect DR by examining the candidate sub-images which do not correspond to the ONH. For microaneurysms and haemorrhages lesions that appear as dark lesions, the work described in (Akram et al., 2014) provides an approach capable of extracting these lesions.

Moreover, assessment of damaged optic nerve head is used to detect glaucoma. A higher ratio between vertical cup diameter and vertical disc diameter indicates a higher risk of glaucoma. Indeed, several works discuss detecting optic disk and cup based on their brightness (Yin et al., 2012; Cheng et al., 2013). Thus, our mobile CAD system may be extended to detect glaucoma. In addition, it can be extended to detect other anatomical structures (Chakravarty and Sivaswamy, 2017; Molina-Casado et al., 2017) or pathologies (Xiayu et al., 2017; Srivastava et al., 2017).

Finally, it is worth pointing out that our proposed processing pipeline can also be implemented on other devices used in ophthalmology (Sharma, 2017), or on handheld embedded systems such as the ones described in (Spitzer and Kayange, 2015; Newman-Casey, 2016).

\section{Conclusion}

In this paper, the Optic Nerve Head (ONH) detection algorithm discussed in (Shahri et al., 2014) was implemented as an app running on Android smartphones. This algorithm uses Radon Transform (RT) to detect the $\mathrm{ONH}$ in retinal fundus images with high accuracy in the presence of high brightness and color variations in such images, in particular when captured by smartphones. The optimization steps taken allow this algorithm to run efficiently on Android smartphones. The experimental results based on fourfundus image databases have shown the effectiveness of the developed app in terms of both the ONH detection accuracy and the execution time of the app. The developed $\mathrm{ONH}$ detection system provides a mobile and cost-effective Computer Aided Diagnosis (CAD) system in ophthalmology which can be deployed in areas where the use of expensive ophthalmology equipment poses a challenge.

\section{CONFLICT OF INTEREST}

The authors declare that they have no conflicts of interest.

\section{REFERENCES}

Akram, M.U., Khalid, S., Tariq, A., Khan, S.A., and Azam F.,2014."Detectionand classification of retinal lesions for grading of diabetic retinopathy", Computers in Biology and Medicine, Vol. 45, pp. 161 -171. DOI: https://doi.org/10.1016/j.compbiomed.2013.11.014

Arunan, A., Pathinarupothi, R. K., Ramesh, M.V., 2016. "A real-time detection and warning of cardiovascular disease LAHB for a wearable wireless ECG device », IEEE Int. Conf. on Biomedical and Health Informatics (BHI), Las-Vegas(USA), pp. 98-101, 24-27. DOI: $10.1109 / \mathrm{BHI} .2016 .7455844$

Ashtari, A.H., Nordin, M.J., Kahaki, S.M.M., 2015."Double Line Image Rotation”, IEEE Trans. on Image Processing, Iss.11, pp. 3370-3385, Vol. 24.DOI: $10.1109 /$ TIP.2015.2440763

Besenczi, R., Szitha, K., Harangi, B., Csutak, A., Hajdu, A., 2015. "Automatic optic disc and optic cup detection in retinal images acquired by mobile phone", $9^{\text {th }}$ Int. Symp.on Image and Signal Processing and Analysis (ISPA), Zagreb, Croatia. DOI: 10.1109/ISPA.2015.7306057

Blanckenberg, M., Worst, C.,Scheffer, C., 2011."Development of a mobile phone based ophthalmoscope for telemedicine", Int. Conf. of the IEEE Engineering in Medicine and Biology Society, Boston, USA.DOI: $10.1109 /$ IEMBS.2011.6091295

Bourouis, A., Feham, M., Hossain, M.A., Zhang, L.,2014. “ An intelligent mobile based decision support system for retinal disease diagnosis", Decision Support Systems, vol. 59, pp. 341-350. DOI:https://doi.org/10.1016/j.dss.2014.01.005

Chakravarty, A., Sivaswamy, J., "Joint optic disc and cup boundary extraction from monocular fundus images", Computer Methods and Programs in Biomedicine, Vol. 147, August 2017, pp. 51-61. DOI: https://doi.org/10.1016/j.cmpb.2017.06.004

Chen, S.W., Wang, X.S., Sato, M., 2014."Uniform Polarimetric Matrix Rotation Theory and Its Applications", IEEE Trans. on Geoscience and Remote Sensing, Iss.8, pp. 4756-4770, Vol. 52.DOI : DOI: 10.1109/TGRS.2013.2284359

Cheng, J., et al., 2013, "Superpixel Classification Based Optic Disc and Optic Cup Segmentation for Glaucoma Screening", IEEE Transactions on Medical Imaging, Vol. 32, No. 6, pp. 1019-1032. DOI: 10.1109/TMI.2013.2247770

Dai, X.B., Shu, H.Z., Luo, L.M., Han, G.N., Coatrieux, J.L., 2010. "Reconstruction of tomographic images from limited range projections using discrete Radon transform and Tchebichef moments", Pattern Recognition, Iss. 3, pp. $1152-1164$, Vol. 43. DOI: https://doi.org/10.1016/j.patcog.2009.07.009

Dash, R., and Majhi, B., 2014, "Motion blur parameters estimation for image restoration", Int. J. for Light and Electron Optics, Iss. 5, pp.1634-1640, Vol. 125. DOI: https://doi.org/10.1016/j.ijleo.2013.09.026

De Marsico, M., Galdi, C., Nappi, M., Riccio, D., 2014."FIRME: Face and Iris Recognition for Mobile Engagement", Image and Vision Computing, Iss.12, pp. 1161-1172, Vol. $32 . \quad$ DOI: https://doi.org/10.1016/j.imavis.2013.12.014

Elloumi, Y., Akil, M., Kehtarnavaz, N., 2017, “ Smartphone captured Retinal Image DataBase », DOI: https://sites.google.com/site/yaroubelloumi/retinal-images.

Elloumi, Y., Akil, M., Kehtarnavaz, N., 2017, "Video demonstration of the Mobile Computer Aided System for Optic Nerve Head Detection", DOI: www.utdallas.edu/ kehtar/Smartphone-ONH.avi.

Gagnon, L., Lalonde, M., Beaulieu, M., Boucher, M.C., 2001."Procedure to detect anatomical structures in optical fundus images", Conf. Med. Imaging: Image Process., San Diego,USA. DOI : 10.1117/12.430999

Gardner, G. G., Keating, D., Williamson, T. H., Elliott, A. T., 1996, "Automatic detection of diabetic retinopathy using an artificial neural network: a screening tool", British Journal of Ophthalmology, vol. 80, pp. 940-944.

Gupta, G., Kulasekaran, S., Ram, K., Joshi, N., Sivaprakasam, M., Gandhi, R., 2017. "Local characterization of neovascularization and identification of proliferative diabetic retinopathy in retinal fundus images", Computerized 
Medical Imaging and Graphics, Volume 55, Pages 124-132. DOI: doi.org/10.1016/j.compmedimag.2016.08.005

Harwood, A.R.G., Revell, A.J., 2017. "Parallelisation of an interactive latticeBoltzmann method on an Android-powered mobile device", Advances in Engineering Software, pp. 38-50, Vol. 104.DOI: doi.org/10.1016/j.advengsoft.2016.11.005

Jadhav, D.V., Holambe, R.S., 2010. "Rotation, illumination invariant polynomial kernel Fisher discriminant analysis using Radon and discrete cosine transforms based features for face recognition", Pattern Recognition Letters, Iss. 9, pp. 1002-1009, vol. 31.DOI: doi.org/10.1016/j.patrec.2009.12.026

Kingston, A., Svalbe, I., 2003. "Mapping between digital and continuous projections via the discrete Radon transform in Fourier space", CSIRO Publishing on Digital Imaging Computing: Techniques and Applications, pp. 263-272, Sydney, Australia. DOI: citeseerx.ist.psu.edu/viewdoc/summary?doi=10.1.1.111.3120

Kingston, A., 2006. "Orthogonal discrete Radon transform over $\mathrm{p}^{\mathrm{n}} \times \mathrm{p}^{\mathrm{n}}$ images", Signal Processing, Iss. 8, pp. 2040 - 2050, Vol. 86. DOI: doi.org/10.1016/j.sigpro.2005.09.024

Kim, T., Kim, J., 2007. "Integration of Code Scheduling, Memory Allocation, and Array Binding for Memory-Access Optimization", IEEE Trans. on Computer-Aided Design of Integrated Circuits and Systems, Iss. 1, pp. 142 - 151, Vol. 26.DOI: 10.1109/TCAD.2006.882639

Kochner, B., Schuhmann, D., Michaelis, M., Mann, G., Englemeier, K.H., 1998."Course tracking and contour extraction of retinal vessels from color fundus photographs: most efficient use of steerable filters for model-based image analysis", Proc. SPIE Med. Imaging, pp. 755761.DOI: $10.1117 / 12.310955$

Lee, D., Kim, Y., Seshadri, V., Liu, J., Subramanian, L., Multu, O., 2013. "Tiered-Latency DRAM: A Low Latency and Low Cost DRAM Architecture", 19th IEEE Int. Symp.On High Performance Computer Architecture (HPCA), Shenzhen (China). DOI: 10.1109/HPCA.2013.6522354

Lee, S., WookJeon, J., 2010."Evaluating Performance of Android Platform Using Native C for Embedded Systems", Int. Conf. on Control, Automation and Systems, Gyeonggi-do, Korea. DOI: DOI: $\underline{10.1109 / I C C A S .2010 .5669738}$

Liang-Hsuan, L., Shang-Lin, C., Shun-Hwa, W., Chueh-Ho, L., Wen-Hsu, S., "Development and clinical application of a computer-aided real-time feedback system for detecting in-bed physical activities", Computer Methods and Programs in Biomedicine,Vol. 147, August 2017, pp. 11-17. DOI: https://doi.org/10.1016/j.cmpb.2017.05.014

Lidong, H., Wei, Z., Jun, W., Zebin, S., 2015.“Combination of contrast limited adaptive histogram equalisation and discrete wavelet transform for image enhancement”, IET Image Processing, Iss. 10, pp. 908 - 915, Vol. 9. DOI: $10.1049 /$ iet-ipr.2015.0150

Mazumdar, B., Mukhopadhyay, D., 2017. "Construction of Rotation Symmetric S-Boxes with High Nonlinearity and Improved DPA Resistivity", IEEE Trans. on Computers, Iss.1, pp. 59-72, Vol. 66. DOI: DOI: $10.1109 / T C .2016 .2569410$

Mitrovic, N., Narayanan, A., Asif, M.T., Rauf, A., Dauwels, J., Jaillet, P., 2016. "On Centralized and Decentralized Architectures for Traffic Applications", IEEE Trans. on Intelligent Transportation Systems, Iss. 7, pp. 1988 - 1997, Vol. 17.DOI: 10.1109/TITS.2016.2519922

Molina-Casado, J. M., Carmona, E.J., Garcia-Feijoo, J., "Fast detection of the main anatomical structures in digital retinal images based on intra- and inter-structure relational knowledge", Computer Methods and Programs in Biomedicine, Vol. 149, October 2017, pp. 55-68. DOI: https://doi.org/10.1016/j.cmpb.2017.06.022

Nacereddine, N., Tabbone, S., Ziou, D., 2015."Similarity transformation parameters recovery based on Radon transform: Application in image registration and object recognition", Pattern Recognition, Iss. 7, pp.22272240, Vol. 48. DOI: https://doi.org/10.1016/j.patcog.2015.01.017

Newman-Casey, P.A., 2016," Portable non-mydriatic device closes gap on tele-glaucoma screening".

Nouvel, B., Rémila, E., 2006. "Incremental and Transitive Discrete Rotations", Lecture Notes in Computer Science (Springer), pp. 199213, vol 4040, Berlin, Germany. DOI: link.springer.com/chapter/10.1007/11774938 16

Prentašić, P., Lončarić, S., 2016."Detection of exudates in fundus photographs using deep neural networks and anatomical landmark detection fusion",
Computer Methods and Programs in Biomedicine, vol. 137, pp. 281-292. DOI :https://doi.org/10.1016/j.cmpb.2016.09.018

Russo, A., Morescalchi, F., Costagliola, C., Delcassi, L., Semeraro, F., 2015."A Novel Device to Exploit the Smartphone Camera for Fundus Photography", Journal of Ophthalmology, Article ID 823139, Volume 2015. DOI:http://dx.doi.org/10.1155/2015/823139

Schmit, H., Thomas, D., 1995. "Array mapping in behavioral synthesis", $8^{\text {th }}$ Int. Symp.on System Synthesis, Cannes, France. DOI: $10.1109 /$ ISSS.1995.520618

Shahri, R.P., M. Tavakoli, N. Kehtarnavaz, 2014.“Computationally efficient optic nerve head detection in retinal fundus images", Biomedical Signal Processing and Control, vol. 11,pp. 63-73.DOI: https://doi.org/10.1016/j.bspc.2014.02.011

Sharma, A.,2017. "Emerging Simplified Retinal Imaging", Pathogenesis and Imaging, Dev Ophthalmol. Basel, Karger, pp. 56-62, vol 60. DOI: $10.1159 / 000459690$

Spitzer, M.S., Kayange, P.C., 2015, "The ZEISS VISUSCOUT 100 Handheld Fundus Camera - A Valuable Tool for Outreach Programs in Rural and Underserved Regions".

Srivastava, R., Duan, L., Wong, D.W.K., Liu, J., Wong, T.Y., "Detecting retinal microaneurysms and hemorrhages with robustness to the presence of blood vessels", Computer Methods and Programs in Biomedicine, Vol. 138, January 2017, Pages 83-91, DOI: https://doi.org/10.1016/j.cmpb.2016.10.017

Sun, M., Tan, G., 2014."Native Guard: Protecting Android Applications from Third-Party Native Libraries", ACM conf. on Security and privacy in wireless \& mobile networks, pp. 165-176, Oxford, UK. DOI: $10.1145 / 2627393.2627396$

Subramanian, L., Lee, D., Seshadri, V., Radtogi, H., Multu, O., 2016. "BLISS Balancing Performance, Fairness and Complexity in Memory Access Scheduling”, IEEE Trans. on Parallel and Distributed Systems, Iss. 10, pp. 3071-3087, Vol. 27.DOI: 10.1109/TPDS.2016.2526003

Svalbe, I., 2011. "Exact, scaled image rotations in finite Radon transform space", Pattern Recognition Letters, Iss. 9, pp. 1415-1420, vol. 32.DOI: https://doi.org/10.1016/j.patrec.2010.06.015

Tolias, Y., Panas, S., 1998. "A fuzzy vessel tracking algorithm for retinal based on fuzzy clustering”, IEEE Trans. Med. Imaging 17, Iss.2, pp. 263 273, Vol. 17.DOI: 10.1109/42.700738

Vianello, A., Chittaro, L., Burigat, S., Budai, R., "MotorBrain: A mobile app for the assessment of users' motor performance in neurology", Computer Methods and Programs in Biomedicine, Vol. 143, May 2017, pp. 35-47. DOI :https://doi.org/10.1016/j.cmpb.2017.02.012

Von Luhmann, A., Wabnitz, H., Sander, T., Muller, K.R., 2016.“M3BA: A Mobile, Modular, Multimodal Biosignal Acquisition architecture for miniaturized EEG-NIRS based hybrid BCI and monitoring", IEEE Trans. on Biomedical Engineering, Iss.99, pp.1199-1210, Vol. 64.DOI: 10.1109/TBME.2016.2594127

Xiayu, X., Wenxiang, D., Ruofan Cao, M.D.A., "An improved arteriovenous classification method for the early diagnostics of various diseases in retinal image", Computer Methods and Programs in Biomedicine, Vol. 141, April 2017, Pages 3-9. DOI: https://doi.org/10.1016/j.cmpb.2017.01.007

Yin, F., et al., 2012," Automated segmentation of optic disc and optic cup in fundus images for glaucoma diagnosis", 25th International Symposium on Computer-Based Medical Systems (CBMS), Italy (Rome).DOI: 10.1109/CBMS.2012.6266344

Yin, S., Xie, Z., Meng, C., Liu, L., Wei, S.,2016."Multibank Memory Optimization for Parallel Data Access in Multiple Data Arrays", 2016 IEEE/ACM Int. Conf. on Computer-Aided Design (ICCAD), Austin, USA.DOI: $10.1145 / 2966986.2967056$

Yu, D., Yang, F., Yang, C., Leng, C., Cao, J., Wang, Y., Tian, J., 2016."Fast Rotation-Free Feature Based Image Registration Using Improved N-SIFT and GMM Based Parallel Optimization", IEEE Trans. on Biomedical $\begin{array}{lllll}\text { Engineering, } \quad \text { Iss. } & 8, \quad \text { pp. } 1653- & 1664, \quad \text { Vol. }\end{array}$ 63.DOI: 10.1109/TBME.2015.2465855

Yuan,G.L., Bakhoda, A.,Aamodt, T. M., 2009. "Complexity Effective Memory Access Scheduling forMany-Core Accelerator Architectures", 42nd Annual IEEE/ACM int. Symp. Microarchitecture, New York, USA.DOI: $\underline{10.1145 / 1669112.1669119}$

Zhang, F., Lian, Y.,2009.“QRS Detection Based on Multiscale Mathematical Morphology for Wearable ECG Devices in Body Area Networks », IEEE 
Trans. on Biomedical Circuits and Systems, Iss.4, pp. 220-228,Vol. 3.DOI: 10.1109/TBCAS.2009.2020093

Zhang, X., et al, 2014, "Exudate Detection in Color Retinal Images for Mass Screening of Diabetic Retinopathy", Medical Image Analysis, Vol. 18, Issue 7, pp. 1026-1043.DOI :https://doi.org/10.1016/i.media.2014.05.004 